\title{
Zwischen Parlamentsvorbehalt und Regierungsdominanz: die wachsende Bedeutung des ungarischen Parlaments im europäischen Integrationsprozess
}

\author{
Jürgen Dieringer
}

In politikwissenschaftlicher Literatur wird immer häufiger abnehmende Steuerungskompetenz der Parlamente behauptet und die These von der „Entparlamentarisierung“ vertreten. Unter anderem sei dies dem europäischen Integrationsprozess geschuldet. Regierungen dominierten den Zugang zur europäischen Ebene, die Parlamente seien in der Gewaltenverschränkung eingeklemmt, politisch der Regierung verbunden, kein einheitlicher Akteur und zudem mangels Information, Sachverstand und institutioneller Kapazität strukturell unterlegen. Ein Blick in die Empirie nationaler Parlamente fördert allerdings eher Divergenz als Konvergenz zu Tage. Der Wandel institutioneller Ordnungen kann somit nicht singulär als (automatisierte) Reaktion auf europäische Entscheidungsprozesse definiert werden. Stattdessen ist von einer gewissen Adaptionsbreite auszugehen, die nationale Unterschiede zulässt, aufgrund nationaler Besonderheiten diese Unterschiede gar zwangsläufig generiert.

Diese Zusammenhänge sollen im Folgenden für Ungarn herausgearbeitet werden. Ziel ist es, die Anpassungsleistungen der Budapester Volksvertretung an den europäischen Integrationsprozess nachzuzeichnen und die Triebfedern für institutionellen Wandel freizulegen.

\section{Entparlamentarisierung und Re-Parlamentarisierung im Integrationsprozess}

„Entmachtung “1, „Entgrenzung“, das Aufkommen paralleler Machtzentren ${ }^{2}$ sind Stichworte, mit denen die neuerliche Diskussion um den „Niedergang“ der Parlamente geführt wird. Sie gerieten gegenüber der Exekutive ins Hintertreffen ${ }^{3}$. Die „gouvernementale Parlamentskontrolle“ etwa wird von Eberhard Schuett-Wetschky als „Missverständnis“ charakterisiert" . Die Parlamente seien eher schwerfällig, zu heterogen, kumulierten zu wenig Sachverstand, als dass sie den Regierungen machtpolitisch in die Augen schauen könnten. Die Europäische Integration ist daran nicht unschuldig. Sie verstärkt den identifizierten Trend einer „Entparlamentarisierung“, bevorzugt die „Gatekeeper“, die nationalen Regierungen als eigentlichen politischen pouvoir constituant der EU.

1 Hans H. Klein, Entmachtung der Parlamente, in: FAZ in 29. November 2004, S. 8.

2 Klein zufolge ist dies problematisch, wenn der Exekutive vom Gesetzgeber Normsetzungskompetenz verliehen wird, sich das Parlament selbst entmachtet („Entparlamentarisierung durch Parlamentsentscheid“), die „Quintessenz des demokratischen Prinzips diffundiert“, ebenda.

3 Vgl. umfassend Andreas Maurer, National Parliaments in the European Architecture: From Latecomers towards Permanent Institutional Change?, in: ders. / Wolfgang Wessels (Hrsg.), National Parliaments on Their Ways to Europe, Baden-Baden 2001, S. $27 \mathrm{ff}$.

4 Eberhard Schuett-Wetschky, Gouvernementale Parlamentskontrolle? Politische Führung, Regierungsmehrheiten und das Verhältnis von Parlament und Regierung, in: Everhard Holtmann I Werner J. Patzelt (Hrsg.), Kampf der Gewalten?, Wiesbaden 2004, S. 22. 
Der Versuch der Parlamente, Kompetenzverluste auszugleichen, führt in ein Dilemma: Ein Bedeutungsgewinn nationaler Parlamente kollidiert mit dem exekutiv-föderalen, konkordanzdemokratischen politischen System der EU ${ }^{5}$. Die Logik der institutionellen Ordnung der EU sieht die nationalen Parlamente nur in begleitender, mitwirkender, weniger in einer gestaltenden, normsetzenden Rolle: „Das Fazit ist genauso simpel wie ausweglos: Effektive national-parlamentarische Legitimation und exekutivföderale Institutionenordnung konterkarieren sich." 6

Das Problem ist strukturell und machtpolitisch gelagert. Während im nationalstaatlichen Politikprozess Parlamente Gesetzgebung unmittelbar initiieren und vor allem Gesetzentwürfe der Regierung modifizieren können, sind so genannte Unionsvorlagen ein zwischen den Regierungen und zwischen Rat und Kommission beziehungsweise Europäischem Parlament geschlossener Kompromiss, oft eine Paketlösung. Die Antwortalternativen der nationalen Parlamente reduzieren sich im Primärrecht auf „Ja“ oder „Nein“, im Sekundärrecht oft auf technische Fragen. Ein „Nein“ ist unwahrscheinlich: In gewaltenverschränkenden Systemen stützt die Parlamentsmehrheit meist die Regierung ${ }^{7}$; Regierung und Parlamentsmehrheit sind als „Regierungsmehrheit“ verschmolzen ${ }^{8}$; Regierungsmitglieder verfügen parallel über einen Sitz im Parlament. Auch bestehen intermediäre Verbindungslinien, etwa über die Parteizugehörigkeit. Die Ablehnung einer von der Regierung auf europäischem Parkett mitbeschlossenen Unionsvorlage ex post grenzte deshalb an politische Selbstzerfleischung, ist demnach unwahrscheinlich. Nur ex ante kann das Parlament beziehungsweise die Mehrheit Vorbehalte einbringen.

Trotz identischer Ausgangslage liefert die Empirie eine erstaunliche Varianz ${ }^{9}$. Neben den aufgrund der Eingangshypothese zu erwartenden Verlierern stehen langsame Anpasser, manche Parlamente pflegen gar den „institutionellen Konter“, die „Hinterbänkler des

5 Vgl. Philipp Dann, Parlamente im Exekutivföderalismus. Eine Studie zum Verhältnis von föderaler Ordnung und parlamentarischer Demokratie in der Europäischen Union, Berlin 2004, S. 269. Das Verhältnis von Parlamentarismus und Europäischer Integration wirft dabei grundlegende Fragen von Demokratie und Legitimation auf, vgl. hierzu stellvertretend Winfried Steffani I Uwe Thaysen (Hrsg.), Demokratie in Europa: Zur Rolle der Parlamente, Opladen 1995.

6 Philipp Dann, a.a.O. (Fn. 5).

7 Dies ist grundlegend anders bei Minderheitsregierungen, wie gelegentlich zum Beispiel in Dänemark, vgl. Finn Laursen, The Danish Folketing and its European Affairs Committee, in: Andreas Maurer / Wolfgang Wessels, a.a.O. (Fn. 3), S. 99 - 116. Hier wird das Parlament zu einem starken Spieler.

8 Eberhard Schuett-Wetschky, a.a.O. (Fn. 4), S. 18. Bei Minderheitsregierungen kommt zwar eine Parlamentsmehrheit für die Regierung und das Regierungsprogramm zustande, jedoch keine Regierungsmehrheit in dem Sinne, als es zwischen Regierung, Partei und Parlament zu einer hierarchisch strukturierten Verschmelzung käme, vgl. ebenda, S. 32.

9 Vgl. unter anderem Philip Norton (Hrsg.), National Parliaments and the European Union, London 1996; Andreas Maurer / Wolfgang Wessels (Hrsg.), a.a.O. (Fn. 3); Jürgen Dieringer / Andreas Maurer / Enikö Györi (Hrsg.), Europäische Entscheidungen kontrollieren. Nationale Parlamente im Ost-West-Vergleich, Dresden 2005. 
Integrationsprozesses "10 schlagen zurück ${ }^{11}$. Woher rühren diese Unterschiede? Wie können Parlamente überhaupt auf die „Entparlamentarisierung“ reagieren?

Einerseits legt die Pfadabhängigkeit politischen Wandels nahe, dass Reformen inkrementalistischer, nicht radikaler Natur sind ${ }^{12}$. Andererseits dürfte das Vorhandensein von Vetospielern und Vetopunkten, sofern sich eine Gelegenheit ergibt, von Zeit zu Zeit auch drastischen Wandel zulassen. Eher inkrementalistisch wäre die Strukturierung des bei den Parlamenten eingehenden europapolitischen Materials analog zur Bedeutsamkeit, eventuell auch die Modernisierung der institutionellen Gestalt durch Stärkung der Europaausschüsse. Verbesserter Informationszugang des Parlaments kann - methodisch „weich“ - entweder durch „Verhandlung“ mit der Regierung, durch Überzeugung erzielt werden oder aber „hart“ durch rechtliche Regelung unter Ausspielung einer Machtposition. Der Versuch der Parlamentarier, die Regierungskontrolle durch die Einführung eines de jure oder de facto bindenden Mandats zu gewährleisten, erfordert meist eine Vetoposition.

\section{Parlamente in Mittel- und Osteuropa}

Die Verfassungen der sozialistischen Staaten wiesen den Parlamenten eine zentrale Rolle im Regierungssystem $\mathrm{zu}^{13}$. Allerdings wurden die Volksvertretungen in der politischen Praxis funktional entleert und politisch marginalisiert ${ }^{14}$. Eine wirkliche Volksvertretung fand nicht statt, die Tätigkeit war demokratisch nicht legitimiert. Géza Kilényi und Vanda Lamm stellten für Ungarn im Jahre 1988 fest:

„The Constitution of the Hungarian People's Republic specifies that Parliament is the highest organ of sovereign power and popular representation (...). This is all very well, on paper. But, if there is a body in Hungary today whose legislatively declared functions and actual status in the system of political institutions show very few points of coincidence it is certainly the Parliament." 15

10 Tapio Raunio / Simon Hix, Backbenchers Learn to Fight Back: European Integration and Parliamentary Government, in: Klaus H. Goetz / Simon Hix (Hrsg.), Europeanised Politics? European Integration and National Political Systems, in: West European Politics, Special Issue, 23. Jg. (2001), S. 142.

$11 \mathrm{Zu}$ beobachten ist dies in der Konventsdeliberation. Im Verfassungsvertrag wurden die Befugnisse der nationalen Parlamente gestärkt, vgl. Jürgen Dieringer, Entparlamentarisierung oder Renaissance der Volksvertretungen? Zur Rolle nationaler Parlamente im europäischen Integrationsprozess, in: Klaus Beckmann / Jürgen Dieringer / Ulrich Hufeld (Hrsg.), Eine Verfassung für Europa, 2. Auflage, Tübingen 2005, S. $251 \mathrm{ff}$.

12 Vgl. Dionyssis Dimitrakopoulos, Incrementalism and Path Dependance: European Integration and Institutional Change in National Parliaments, in: Journal of Common Market Studies, 39. Jg. (2001), S. $405 \mathrm{ff}$.

13 Vgl. Georg Brunner / Boris Meissner (Hrsg.), Verfassungen der kommunistischen Staaten, Paderborn u.a. 1979, S. 15.

14 Gabriella Ilonszki, From Marginal to Rational Parliaments: A Central European Regional View, in: Attila Ágh / Gabriella Ilonszki (Hrsg.), Parliaments and Organized Interests: The Second Steps, Budapest 1996, S. 423.

15 Géza Kilényi / Vanda Lamm, Parliament and Government in a one-party system, Budapest 1988, S. 57. 
Im Systemwechsel wurden die Parlamente vom stillen Beobachter zu „central sites of democratization“16. Sogar von „Überparlamentarisierung“ wurde gesprochen ${ }^{17}$. Im „Gesetzgebungssturm“ der Transformationsjahre fungierten die Parlamente als „Gesetzfabriken“. Sie waren aktive "policy-maker"18 und kompensierten instabile Kabinette. Die Arbeitsbelastung der Parlamente war während dieser Zeit enorm. Arthur Benz leitet aus der zentralen Position der Parlamente während der Systemtransformation die These ab, dass sich die Parlamente der EU-Neumitglieder als Vorreiter bei der Rückeroberung verlorenen Terrains erweisen könnten ${ }^{19}$. Gleichwohl läßt der Beitrittsprozess zur EU aufgrund seiner Exekutivlastigkeit etwas anderes vermuten:

„[ECE countries] have delayed the transition to the parliamentary and society-centred stages, including the organization of the necessary networks and institutions (...). They have suffered from prolonged governmental dominance in the EU integration process, and so far the participation of both the parliament and population in this process has remained very weak and marginal. Therefore, the policy-making process concerning the EU has still been over-concentrated in the hands of central government (...).“20

Auch andere Argumente sprechen gegen die osteuropäischen Legislativen: Wählerentscheidungen sind volatil, es kommt zu einem permanenten Austausch der Abgeordneten. Diese sind unerfahren, mit europäischen Materien kaum vertraut. Mangelnde Sprachkenntnisse, Desinteresse aufgrund schlechter Bezahlung, Tanz auf mehreren Hochzeiten sind Probleme, mit denen die Parlamente in der Europapolitik zu kämpfen haben. Attila Ágh spricht hier zutreffend vom „opportunity-capacity paradox“21. Die Gelegenheit wäre da, die Kapazitäten aber sind begrenzt. Zusammenfassend gilt: „The new situation after the accession is both easier and more difficult at the same time. "22 Sie ist einfacher, weil der „Gesetzgebungssturm" beendet und der acquis communautaire übernommen ist, die Arbeitslast in dieser Hinsicht abnimmt, und sie ist komplizierter, weil „Europa“ seit dem Beitritt noch weiter in nationalstaatliche Strukturen penetriert.

16 Vgl. David M. Olson, The Centrality of Post-Communist Parliaments of Central Europe in Democratic System Transition and Consolidation, in: Susanne Kraatz / Silvia von Steinsdorff(Hrsg.), Parlamente und Systemtramsformation im postsozialistischen Europa, Opladen 2002, S. 17 - 42; Drago Zajc, A Reconsideration of the Role of the ECE Parliaments in the Process of Europeanisation, in: Máté Szabó (Hrsg.), The Challenge of Europeanisation in the Region: East Central Europe, Budapest 1996, S. 163 - 172.

17 Attila Ágh, Parliamentarization as a Region-Specific Way of Democratization in East Central Europe, in: Susanne Kraatz / Silvia von Steinsdorff, a.a.O. (Fn. 16), S. 43.

18 Radoslaw Zubek, A core in check: the transformation of the Polish core executive, in: Journal of European Public Policy, 8. Jg. (2001), S. 917.

19 Arthur Benz, National parliaments in the European Union - West European experiences and lessons for the new Member States, in: Attila Ágh (Hrsg.), Post-Accession in East Central Europe. The Emergence of the EU 25, Budapest 2004, S. $58 \mathrm{f}$.

20 Attila Ágh, Europeanization of policy-making in East Central Europe: the Hungarian approach to EU accession, in: Journal of European Public Policy, 6. Jg. (1999), S. 845.

21 Attila Ágh, The Role of the ECE Parliaments in the EU integration, in: Attila Ágh, a.a.O. (Fn. 19), S. 84

22 Ebenda, S. 86. 


\section{Grundlagen des ungarischen Parlamentarismus}

Das parlamentarische Regierungssystem Ungarns ist unikameral organisiert. Das Parlament ist im Verhältnis zur Bevölkerungszahl des Landes (circa zehn Millionen) mit 386 Abgeordneten sehr groß. Wahlfunktionen bestehen gegenüber dem Staatspräsidenten und dem Ministerpräsidenten, nicht aber gegenüber den Ministern. Das Misstrauensvotum ist konstruktiv, eine Inkompatibilität von Amt und Mandat nicht gegeben. Gesetze können vom Staatspräsidenten, der Regierung, einem Ausschuss oder einem Abgeordneten eingebracht werden. Das Vetorecht des Staatspräsidenten ist lediglich suspensiv und kann mit einfacher Mehrheit überstimmt werden. Die Kontrollfunktion der Abgeordneten wird wie üblich mit Hilfe des Interpellationsrechts und Untersuchungsausschüssen ausgeübt.

Die ungarische Verfassung stellt das Parlament als „höchstes Organ der Staatsmacht und der Volksvertretung“ (Art. 19[1]) ins Zentrum des politischen Entscheidungsgefüges. Nur ihm obliegt verfassungsändernde Reform. Die Volksvertretung „(...) legt die Organisation, die Richtung und die Bedingungen des Regierens fest“ (Art. 19[2]). Der Ministerpräsident verfügt de jure über keine Richtlinienkompetenz. Die formal starke Stellung des Parlaments wird allerdings begrenzt durch die de facto gesteigerte Bedeutung des Regierungschefs in der ungarischen „Kanzlerdemokratie“23. Die starken Persönlichkeitsprofile der Ministerpräsidenten ${ }^{24}$ haben das Parlament „domestiziert“. Gestärkt wird die Legislative gegenüber der Exekutive hingegen durch die hohe Anzahl an so genannten Zweidrittelgesetzen ${ }^{25}$, die die Opposition in die Rolle eines Vetospielers versetzen. Über 30 Gesetzgebungsbereiche sind hiervon betroffen ${ }^{26}$.

Die Regierungen der letzten 17 Jahre fußten immer auf einer gesicherten parlamentarischen Mehrheit. Teilweise wurden „Minimum winning-Koalitionen“ gebildet, aber auch „oversized coalitions“ sind vorgekommen. Eine Minderheitsregierung wurde stets vermieden.

23 Vor allem gegenüber dem formal nicht unbedeutenden Staatspräsidenten konnten sich die Ministerpräsidenten beziehungsweise die Regierung als Ganze - mit Unterstützung der Verfassungsrechtsprechung - formale Kompetenzen und informelle Machtpotentiale sichern, vgl. Jürgen Dieringer, Staatspräsident Árpád Göncz - Wegbereiter der ungarischen „Kanzlerdemokratie“ wider Willen, in: Südosteuropa, 53. Jg. (2005), S. $272 \mathrm{ff}$.

24 Mit Ausnahme der „Übergangspremiers“ Péter Boross (Herbst 1993 bis Frühjahr 1994) und Péter Medgyessy (2002 bis 2004).

25 Bei den „Zweidrittelgesetzen“, die ein Ergebnis des Interessensausgleichs im Verfassungsgebungsprozess am Runden Tisch sind, handelt es sich um Regelungsbereiche, in denen im Parlament eine Zweidrittelmehrheit erforderlich ist. Diese sind über die gesamte Verfassung verstreut. Die erste demokratische Regierung betrachtete diese Zweidrittelgesetze als Hemmnis und einigte sich mit der damals größten Oppositionspartei auf ein Bündnis. Dieser „Antall-Tölgyessy-Pakt“ diente 1990 dazu, verfassungsändernde Gesetze durchs Parlament zu bringen beziehungsweise die Zweidrittelmehrheit in Politikfeldern zu erreichen, in denen die Zweidrittelregelung einschlägig ist. Der SZDSZ wurde für die Zustimmung mit dem Amt des Staatspräsidenten „,entschädigt“.

26 András Körösényi, Das politische System Ungarns, in: Wolfgang Ismayr (Hrsg.), Die politischen Systeme Osteuropas, Opladen 2002, S. 313. 


\begin{tabular}{|c|c|c|c|c|c|}
\hline Legislatur & 1990 bis 1994 & 1994 bis 1998 & 1998 bis 2002 & 2002 bis 2006 & 2006 bis (2010) \\
\hline Parteien & $\begin{array}{c}\text { MDF-KDNP- } \\
\text { FKGP* }^{*}\end{array}$ & MSZP-SZDSZ & $\begin{array}{c}\text { FIDESZ-FKGP- } \\
\text { MDF }\end{array}$ & MSZP-SZDSZ & MSZP-SZDSZ \\
\hline Charakter & $\begin{array}{l}\text { oversized } \\
\left({ }^{*} \text { minimum- }\right. \\
\text { winning })\end{array}$ & oversized & oversized & $\begin{array}{l}\text { minimum- } \\
\text { winning }\end{array}$ & $\begin{array}{l}\text { minimum- } \\
\text { winning }\end{array}$ \\
\hline $\begin{array}{l}\text { Mehrheits- } \\
\text { verhältnisse }\end{array}$ & $\begin{array}{l}\text { zunächst große, } \\
\text { dann knappe } \\
\text { Mehrheit }\end{array}$ & $\begin{array}{l}\text { Zweidrittel- } \\
\text { mehrheit }\end{array}$ & $\begin{array}{l}\text { zunächst große, } \\
\text { dann schrump- } \\
\text { fende Mehrheit }\end{array}$ & $\begin{array}{l}\text { knappe, } \\
\text { aber stabile } \\
\text { Mehrheit }\end{array}$ & $\begin{array}{l}\text { knappe, } \\
\text { aber stabile } \\
\text { Mehrheit }\end{array}$ \\
\hline \multicolumn{6}{|c|}{$\begin{array}{l}\text { * Die FKGP trat } 1992 \text { aus der Regierung aus; } \\
\text { FIDESZ: Bund Junger Demokraten (bürgerlich-konservativ); FKGP: Unabhängige Kleinlandwirtepar- } \\
\text { tei (agrarisch-rechtspopulistisch); KDNP: Christlich-Demokratische Volkspartei (christlich-konserva- } \\
\text { tiv); MDF: Ungarisches Demokratisches Forum (bürgerlich); MSZP: Ungarische Sozialistische Partei } \\
\text { (sozialdemokratisch); SZDSZ: Bund Freier Demokraten (liberal). } \\
\text { Quelle: Eigene Darstellung. }\end{array}$} \\
\hline
\end{tabular}

Formal und strukturell scheint das ungarische Parlament als Ganzes demnach gut für die Kontrolle europäischer Politik geeignet zu sein. Stellung im System, Größe, Vetoposition der Opposition sprechen für die Volksvertretung. Falls die guten strukturell-formalen Prädispositionen sich empirisch nicht in Parlamentsmacht umsetzen lassen, müssen Erklärungen in „weicheren" Faktoren zu suchen sein.

\section{Das ungarische Parlament im Integrationsprozess}

Die Organisation der Europäisierung im ungarischen Parlament verlief in drei Schritten: erstens die institutionelle Anpassung; zweitens die Verbesserung der Regierungskontrolle das „scrutiny system“-, bestehend aus verbessertem Informationszugang und der Möglichkeit, die Standpunkte des Parlaments für die Regierung in deren Handeln im Rat der EU verbindlich zu machen; drittens die Konstituierung des Parlaments als Akteur auf europäischer Ebene, um so die „Gatekeeper-Position“ der Regierung zu relativieren sowie Information und Expertise zu verbessern.

Das ungarische Parlament hat sich bereits auf europäischer Ebene konstituiert. Der Europaausschuss arbeitet in der Konferenz der Europaausschüsse (COSAC) mit, und das Parlament eröffnete ein Verbindungsbüro in Brüssel. Damit ist der direkte Zugang zu europäischen Entscheidungsträgern gesichert, die Informationsbeschaffung optimiert. Ob sich hieraus eine Umgehungsstrategie entwickeln könnte, um sich notfalls auch gegen die eigene Regierung positionieren zu können, ist bisher Spekulation. Hier bedarf es der Hebung weiterer, belastbarer Daten. Deshalb sollen hier lediglich die Entwicklung des Ausschusswesens und die Regierungskontrolle bewertet werden.

\subsection{Die Entwicklung des Europaausschusses und des Großausschusses (Nagybizottság)}

Die institutionelle Anpassung betrifft die Etablierung eines Europaausschusses und die Verankerung der Thematik Europäische Integration in den anderen Ausschüssen. Der erste, nichtständige ungarische Ad hoc-Europaausschuss des Jahres 1991 diente der Organisation 
von institutionalisierten Kontakten zur Parlamentarischen Versammlung des Europarates. Mitglied waren acht Parlamentarier, ein Mitglied pro Fraktion sowie zwei Fraktionslose, durchaus hochrangige Politiker ${ }^{27}$. Bereits ein Jahr später war die EG der entscheidende Bezugspunkt. Gegründet wurde ein gemeinsamer Ausschuss mit dem Europäischen Parlament unter dem Vorsitz von Otto von Habsburg. Als Ergebnis der Arbeit des gemischten Ausschusses kann eine individuelle Sozialisierung und die Aneignung von Sachverstand vermeldet werden ${ }^{28}$. Noch 1992 erlangte der Ausschuss den Status eines Sonderausschus$\operatorname{ses}^{29}$. Die Mitgliederzahl wurde verdoppelt.

1994 wurde der Sonderausschuss in einen ständigen Ausschuss umgewandelt ${ }^{30}$ - ein bedeutender Schritt, weil mit der Statusfrage das Initiativrecht verbunden ist, man nicht auf Stellungnahmen beschränkt bleibt. In dieser Phase wurde der Ausschuss politisiert, Mehrheitsverhältnisse wurden zu einem politischen Faktor. So erklärt sich, dass die Mitgliederzahl - nach langen Diskussionen ${ }^{31}$ - auf 15 verringert wurde, um der neuen Regierungskoalition aus MSZP und SZDSZ eine (Zweidrittel-)Mehrheit zu sichern. In der Folge wurde der Ausschuss kontinuierlich aus- und umgebaut. Einerseits um größenmäßig mit dem EP-Bruderausschuss gleichzuziehen, andererseits weil das Verfassungsgericht generell die Besetzung von Parlamentsausschüssen neu regelte. So schwankte die Mitgliederzahl von 23 (1996) über 26 (1998) und 21 (2002) bis zu aktuell 20 (2007). Der Vorsitz ging jeweils an die Regierungsmehrheit, die immer auch die Mehrheit im Ausschuss stellte.

Mit der Umwandlung in einen ständigen Ausschuss ging eine Vertiefung im Bereich wirtschaftspolitischer Fragen einher. Zudem wurde die gesamte Themenpalette der Integration abgedeckt ${ }^{32}$. Gegenstand war in erster Linie die Umsetzung des Europaabkommens, etwa bei der Kontrolle der Jahresberichte der Regierung. Man leitete Untersuchungen ein, organisierte Interpellationen, initiierte und erstellte Positionspapiere. Es handelt sich also um einen klassischen Querschnittsausschuss, der auch die Koordinierung der Fachausschüsse in Europaangelegenheiten übernimmt.

Im Jahre 2001 wurde der ungarische Europaausschuss beziehungsweise die Befassung mit Europaangelegenheiten im ungarischen Parlament strukturell neu ausgerichtet, die zweite große Reform nach 1994. Die wichtigste Neuerung bestand darin, dass es fortan möglich war, Unterausschüsse in den Fachausschüssen einzusetzen. Damit wurde die Kontrollfunktion des Europaausschusses erheblich gestärkt, da ein direkter Draht zu diesen Unterausschüssen entwickelt wurde, welche über mehr fachspezifische Expertise verfügen.

27 Attila Ágh / László Szarvas / László Vass, The Europeanization of the Hungarian Polity, in: Attila Ágh / Sándor Kurtán (Hrsg.), Democratization and Europeanization in Hungary: The First Parliament (1990-1994), Budapest 1995, S. 221.

28 Jürgen Dieringer / Andrej Stuchlik, Nationale Parlamente und Europa im tschechisch-ungarischen Vergleich, in: Jürgen Dieringer / Andreas Maurer / Enikö Győri, a.a.O. (Fn. 9), S. 136.

29 Európai Közösségi Ügyek Bizottsága, EKÜB, Ausschuss für Angelegenheiten der Europäischen Gemeinschaft.

30 Európai Integrációs Ügyek Bizottsága, EIÜB, Ausschuss für Angelegenheiten der Europäischen Integration.

31 Jürgen Dieringer / Andrej Stuchlik, a.a.O. (Fn. 28), S. 136.

32 Deborah Moss, Europeanization: a case study. The Work of the Committee on European Community Affairs in the Hungarian National Assembly, in: Attila Ágh (Hrsg.), The Emergence of East Central European Parliaments: The First Steps, Budapest 1994, S. 140. 
Die Verkoppelung von Fachausschüssen mit dem Europaausschuss erfolgt unter anderem über die so genannten extended sessions des Europaausschusses, an denen die Vorsitzenden der Europa-Unterausschüsse der Fachausschüsse teilnehmen. Diese finden einmal pro Monat im Beisein des Außenministers statt. Die Größe der Unterausschüsse variiert beträchtlich, wie man auch hinsichtlich ihrer politischen Bedeutung von erheblichen Schwankungen ausgehen kann. Enikö Györi kommt zum Ergebnis, dass einige der Ausschüsse lediglich auf dem Papier bestehen ${ }^{33}$. Empirische Materialien liegen diesbezüglich leider noch nicht vor.

Das Prestige des ungarischen Europaausschusses wuchs offenbar parallel zur verbesserten Kompetenzausstattung. 1994 lautete das Urteil: „schwach, aber mit Potenzial“34. Problematisch war die anfangs hohe ${ }^{35}$, später zumindest nicht niedrige Fluktuation in der Mitgliedschaft, die die Arbeit des Ausschusses erschwerte, da Fachexpertise vor diesem Hintergrund nicht verstetigt werden konnte. Als ab Mai 2003 für ein Jahr Mitglieder des Europaausschusses als Beobachter an der Arbeit des Europäischen Parlamentes teilnahmen, wurde es immer schwerer, überhaupt Sitzungen einzuberufen ${ }^{36}$. Dieses Problem ist seit dem Beitritt gelöst, da Mitgliedschaft im nationalen Parlament und Abgeordnetenstatus im EP getrennt wurden.

Die politische Bündelung der europäischen Expertise versah in der Beitrittsphase der so genannte Großausschuss (Európai Uniós Nagybizottság). Seine Struktur lehnte sich stark an das finnische Modell an. Teilnehmer waren der Vorsitzende des Europaausschusses, der Ministerpräsident, der Außenminister, nach Bedarf einzelne Fachminister, der Parlamentspräsident, die Fraktionsvorsitzenden, der Vorsitzende des Auswärtigen Ausschusses und der Vorsitzende des Verfassungs- und Justizausschusses. Der Großausschuss formulierte das übergreifende nationale Interesse. Mit dem Beitritt zur EU wurde er aufgelöst.

\subsection{Regierungskontrolle und Parlamentsvorbehalt}

Die Kontrolle der Regierung in Europaangelegenheiten ergab sich lange aus allgemeinen Normen und Instrumenten der parlamentarischen Kontrolle. Bis kurz vor dem Beitritt Ungarns zur EU war die Parlamentsverordnung 10/1993. (III.) Ogy. einschlägig. Hier wurde der Regierung im einjährigen Rhythmus ein Bericht über den Stand der Integrationsfähigkeit abverlangt. Die Parlamentarier sollten zudem Gelegenheit erhalten, einmal jährlich im Rahmen einer Plenardebatte Regierungsmitglieder zu europapolitischen Themen zu befragen. Mit dem Beitrittsprozess wurde ein eigenständiger Text ausgearbeitet. Seit der Mitgliedschaft in der Europäischen Union weist das Gesetz LIII/2004 in Artikel 1 den Ausschüssen im Allgemeinen und dem Europaausschuss im Besonderen die Aufgabe zu, die Rechte des Parlaments gegenüber der Regierung wahrzunehmen. Art. 1(2) etabliert gar ein Entscheidungsrecht des Ausschusses.

33 Enikö Györi, A Nemzeti Parlamentek és az Európai Unió, Budapest 2004.

34 Attila Agh, a.a.O. (Fn. 32), S. 78.

35 Gabriella Ilonszki, Parlament az Európai Unióban és Magyarországon, in: Politikatudományi Szemle, 7. Jg. (1998), S. 74.

36 Enikö Gyơri, Hogyan őrizheti meg szuverenitását az Országgyűlés az EU-csatlakozás után?, Manuskript, 2003, S. 5. 
Der Text basiert auf einem von einer Arbeitsgruppe ${ }^{37}$ ausgearbeiteten Vorschlag, der im Großausschuss verhandelt wurde und den Auftrag der Verfassungsmodifikation LXI/2002 umsetzt, die Mitwirkung des Parlaments in Europaangelegenheiten generell gesetzlich zu regeln. Die Notwendigkeit der Kompromissfindung führte dazu, dass die Parlamentskontrolle in Angelegenheiten der Europäischen Integration gar in der Verfassung festgeschrieben und einer Zweidrittelmehrheit unterworfen wurde. ${ }^{38}$ Art. 35A bestimmt, dass das entsprechende Einzelgesetz auszuarbeiten und mit Zweidrittelmehrheit zu beschließen sei, die Regierung Transparenz zu schaffen habe über den exekutiven Entscheidungsmechanismus und dessen institutionelle Struktur.

Das aus der Verfassungsbestimmung resultierende Einzelgesetz rekurriert in Artikel 1 allgemein auf die Kontrollrechte des Parlaments in Europaangelegenheiten und die Konsultations- und Informationsrechte der Volksvertretung. Sämtliche europäischen Gesetzesvorhaben mit Beteiligung der Regierung sind dem Parlament unmittelbar nach Eingang in der nationalen Arena zuzuleiten. Das Parlament hat zudem das Recht, ergänzende Materialien jederzeit anzufordern. Die Regierung stellt fest, welche Rechtstexte in den Gestaltungsbereich des Parlaments fallen. Hierzu zählen insbesondere Angelegenheiten, die nach Maßgabe der ungarischen Verfassung einer qualifizierten Mehrheit (hier: einer Zweidrittelmehrheit) unterliegen, die Grundrechte beziehungsweise Grundpflichten und deren Garantie betreffen, schließlich all jene Angelegenheiten, die einen Konflikt mit bestehenden Rechtstexten schaffen. Der Regierung wird aufgetragen, die Materialien dergestalt vorzustrukturieren, dass ersichtlich wird, welche Gegenstände von besonderer Bedeutung für das Land sind, um eine Debatte hierüber sicherzustellen. Der Feststellung der besonderen Bedeutung für das Land und der Zuweisung in den Gestaltungsbereich des Parlaments ist eine Begründung beizufügen.

Parlamentarische Rechte in der Phase des „downloadens“ europäischer Projekte werden ergänzt um Rechte bei der Erstellung der Regierungsposition. Hierzu dienen zuallererst die Informationsrechte bei der Festlegung einer ungarischen Verhandlungsposition. Diese betreffen das Verfahren auf europäischer Ebene, den Zeithorizont, die Ziele, die die Regierung im angesprochenen Prozess verfolgt, und die Begründung für das Vorhaben, schließlich die Rechtsetzungsnotwendigkeiten, die sich für das Parlament ergeben werden. Die Regierung ist ihrerseits berechtigt, das Parlament ex ante zu konsultieren. Diese Informationsrechte können von Fall zu Fall ergänzt beziehungsweise spezifiziert werden um eine Folgenabschätzung insbesondere ökonomischer und budgetärer Natur sowie um die Positionen der europäischen Organe und anderer Mitgliedstaaten soweit bekannt. Handelt es sich um Fragen von strategischem Interesse für das Land, kann der Parlamentspräsident den Ministerpräsidenten nach Art. 7 vor Sitzungen des Europäischen Rates verpflichten, den Vorsitzenden der Parlamentsfraktionen, den Mitgliedern des Europaausschusses sowie den Vorsitzenden des Ausschusses für Verfassungsfragen, des Auswärtigen Ausschusses und an-

37 Beteiligt waren die Regierung, das Parlament, Parteien und unabhängige Sachverständige.

38 Der Wortlaut: „UVerf, Art. 35/A. (1) Az európai integrációval összefüggő ügyekben az Országgyűlés vagy bizottságai ellenőrzési jogkörének, az Országgyűlés és a Kormány között folytatott egyeztetésnek, továbbá a Kormány tájékoztatási kötelezettségének részletes szabályairól a jelenlévő országgyűlési képviselők kétharmadának szavazatával elfogadott törvény rendelkezik.

(2) A Kormány az Országgyűlés részére megküldi azokat a javaslatokat, amelyek az Európai Unió kormányzati részvétellel működő intézményeinek döntéshozatali eljárásában napirenden szerepelnek." 
deren auf Initiative des Präsidenten eingeladenen Vorsitzenden weiterer Ausschüsse oder Unterausschüsse Rede und Antwort zu stehen. Die Informationspflicht bei strategischen Fragen gilt generell und betrifft auch eine mündliche Auskunftspflicht des Ministerpräsidenten über die Ergebnisse der Sitzungen des Europäischen Rates. Des Weiteren muss die Regierung dem Plenum einen Jahresbericht über die Folgen der Mitgliedschaft Ungarns in der Europäischen Union liefern.

Die Frage der Bindungswirkung von Parlamentsbeschlüssen, die so genannte Vorbehaltsregelung, wurde in Ungarn lange diskutiert. Die jeweilige Regierungsfraktion konnte einen Beschluss zugunsten einer starken Bindung aber stets verhindern, während die generelle Kritik am Fehlen einer solchen naturgemäß jeweils von der Opposition stammte ${ }^{39}$. Nur sie war an einer effektiven Kontrolle der Regierung interessiert, nicht hingegen die Regierungsmehrheit. Selbst die Parlamentspräsidentin Katalin Szili (MSZP) wandte sich bei der Diskussion um ein entsprechendes Einzelgesetz 2003/2004 gegen ein bindendes Mandat und damit gegen eine Bedeutungssteigerung der Institution, der sie vorsitzt. Sie wollte verhindern, dass der Regierung im europäischen Verhandlungspoker die Hände gebunden werden.

Die neue Regelung sieht folgendermaßen aus: Erstellt die Regierung auf Verlangen des Parlaments eine Folgenabschätzung und ein Papier mit den Positionen der europäischen Organe und anderer Mitgliedstaaten im oben beschriebenen Verfahren, kann das Parlament in einem angemessenen Zeitraum eine Stellungnahme verfassen, in der die zentralen Punkte der Parlamentsmeinung dargelegt werden. Vor der entscheidenden Ratssitzung kann der zuständige Minister oder Staatssekretär angehört werden. Er hat die Verhandlungsposition der Regierung darzulegen. Diese Position hat, sofern eine Parlamentsstellungnahme oder Parlamentsresolution vorliegt, auf dieser zu fußen. Dies gilt bevorzugt für Fragen, die nach Maßgabe der ungarischen Verfassung einer qualifizierten Mehrheit unterliegen. Hier darf die Regierung vom Standpunkt des Parlaments nur in „begründeten Fällen“ (Art. 4[5]) abweichen. Ist das Parlament nicht in der Lage oder willig, in der durch den europäischen Gesetzgebungsprozess definierten Zeitspanne eine Position zu entwickeln, ist die Regierung in ihrer Entscheidung frei. Ändert die Regierung im Verhandlungsprozess auf europäischer Ebene ihre Position, ist dies - zumindest bei signifikanten Änderungen - dem Parlament mitzuteilen und die Änderungen sind zu rechtfertigen. Das Parlament kann hierauf seinen Vorbehalt ebenfalls verändern. Nach einer Entscheidung im Rat ist das nationale Parlament in jenen Fällen über den Beschluss zu informieren, in denen das Parlament eine Stellungnahme abgegeben hat beziehungsweise wenn das Parlament dies verlangt. In ZweidrittelAngelegenheiten kann das Parlament die Rechtfertigung der Regierung zurückweisen. Dies hat allerdings nur politische Konsequenzen, keine rechtlichen.

Die getroffene Regelung für den gesetzgeberischen „Normalfall“ enthält zwar kein bindendes Mandat, geht aber über die vorherige Lösung weit hinaus, indem sie die Regierung - ganz nach österreichischem Vorbild - zwingt, die Nichtberücksichtigung der Meinung des Parlaments zu begründen. Herausragend ist neben der im Vergleich zu anderen Parlamenten auf dem Papier doch relativ starken Bindungswirkung die Tatsache, dass dem Europaausschuss Letztentscheidungsrecht zugestanden wurde. Er kann demnach an Stelle des Plenums entscheiden und wird zu einer Art „Mini-Parlament“, auch in der Frage der Stel-

39 Vgl. zum Beispiel Nóra Chronowski, Az Országgyűlés és a kormány integrációs ügyekkel kapcsolatos együttműködésének szabályozásáról, in: Magyar Közigazgatás, 53. Jg. (2003), S. 535. 
lungnahme des Parlaments gegenüber der Position der Regierung. Dies erhöht das Prestige des Ausschusses und verbessert die Effizienz des Gesamtparlaments. Einschränkend muss bemerkt werden, dass die parlamentarische Mandatierung der Regierung für EU-Ministerratsentscheidungen in Ungarn nicht ganz so systematisch und generell erfolgt wie in manch anderen Ländern. Ein Mandat erfolgt nur dann, wenn dies von der Regierung nachgefragt wird oder der Integrationsausschuss beziehungsweise eines seiner Mitglieder ein entsprechendes Mandat verlangt ${ }^{40}$.

Unabhängig von der Regierungsaktivität ist das Parlament in Artikel 9 aufgerufen, die Vorlagen der Europäischen Union auf ihre Kompatibilität mit dem Subsidiaritätsgrundsatz abzugleichen und entsprechend Positionen festzulegen. Weitere Vorbehaltsrechte des Parlaments ergeben sich hinsichtlich der Nominierung ungarischer Kandidaten für hohe Ämter in Brüssel ${ }^{41}$. Hier schreibt Art. 8[2] ein Anhörungsrecht der Volksvertretung fest.

\section{Re-Parlamentarisierung durch EU-Mitgliedschaft?}

Formal hat das ungarische Parlament eine beachtliche Wandlung durchgemacht. Europaangelegenheiten gewannen stets an Bedeutung, die institutionellen Strukturen wurden immer wieder angepasst, Regierungskontrolle und Informationszugang nachhaltig verbessert. Dass Sachlogik als Begründungszusammenhang für die Anpassungsleistungen ausreicht, darf bezweifelt werden: Sie waren stets politisch definiert. So übertrugen die Regierungen die jeweiligen Mehrheitsverhältnisse auf das Ausschusswesen. Die Opposition hingegen nutzte ihren Zweidrittel-Vetopunkt, um die Kontrollbefugnisse des Parlaments festzuschreiben. Dass dies zu einer im internationalen Vergleich relativ scharfen Parlamentsvorbehaltsregel geführt hat, liegt an der verfahrenen innenpolitischen Lage sich unversöhnlich gegenüberstehender Parteien. Dieser Gegensatz überdeckt einen in der Sache bestehenden Grundkonsens über elementare Fragen der Integration. Ein Fingerzeig der Opposition, man könne die zum Beitritt erforderlichen Anpassungen auch scheitern lassen, war nicht notwendigerweise eine leere Drohung. So muss die Regierung die formale Einschränkung ihrer Entscheidungshoheit in einem Gesamtintegrationspaket schlucken. Folgte der Entparlamentarisierung also eine Re-Parlamentarisierung?

Dass das scharfe Kontrollverfahren in der Praxis zur stumpfen Waffe werden kann, ist sicherlich nicht ungewollt. Die Sicherung der Mehrheitsverhältnisse in den Kontrollgremien ist das Gegengift. Auch hier wirkt die innenpolitische Spaltung und schweißt die Regierungsmehrheit zusammen. Das Parlament ist kein einheitlicher Akteur, sondern zerfällt in der Praxis in Regierungsmehrheit und Opposition. Die parteipolitische Bindung und das Ziel, eine Regierung handlungsfähig im Amt zu halten, erweisen sich als stärker als die Idee des freien Mandats und die mit dem Mandat verbundene Anforderung an den Abgeordneten, die Regierung öffentlich zu kontrollieren. Gewaltenverschränkung sowie die Ämterund Mandatskumulierung sowohl horizontal als auch vertikal verhindern die Ausbildung

40 Verlässliche und generalisierbare Daten sind bisher noch nicht gehoben worden. Der Eindruck beruht auf der Einschätzung von Mitarbeitern des ungarischen Parlaments.

41 Der Kommissar, Richter am Europäischen Gerichtshof sowie am Gericht erster Instanz, außerdem ungarische Mitglieder der Führungsebene des Europäischen Rechnungshofes und der Europäischen Investitionsbank. 
einer starken parlamentarischen corporate identity, was durchaus auch in den Strukturen des parlamentarischen Regierungssystems möglich wäre und zu einer Re-Parlamentarisierung beitragen könnte.

So kann zusammenfassend der Schluss gezogen werden, dass eine Diskrepanz besteht zwischen den Rechtstexten und der politischen Wirklichkeit - nicht neu in Ungarn. Ursächlich sind sowohl Grundparameter des politischen Systems als auch Faktoren der Politischen Kultur. Die Verbindung beider erklärt bestens die Parallelität von Wandel und Beharrung des ungarischen Parlamentarismus vor dem Hintergrund der Teilnahme des Landes an der Europäischen Integration. Eine Veränderung hin zur politischen Stärkung des Parlaments könnten lediglich eine Abnahme der parteipolitischen Durchdringung der Elite beziehungsweise eine Minderheitsregierung initiieren. Beide Varianten sind eher unwahrscheinlich.

\title{
Vertikale Gewaltenteilung: institutionenpolitische Leitidee oder demokratietheoretische Chiffre? Reform westeuropäischer Bundes- und Einheitsstaaten im Vergleich
}

\author{
Florian Grotz
}

Föderalismus als Organisationsprinzip politischer Herrschaft erfreut sich derzeit großer Beliebtheit. ${ }^{1}$ Zwar ist die überwiegende Staatenmehrheit noch immer unitarisch verfasst, doch gibt es eine Reihe von Ländern, die in der jüngeren Vergangenheit eine bundesstaatliche Ordnung eingeführt haben, zum Beispiel Äthiopien, Belgien, Bosnien-Herzegowina oder Südafrika. Hinzu tritt eine weit höhere Zahl von Staaten, die eine Abkehr vom unitarischen Prinzip vollzogen haben oder zumindest anstreben. Dies gilt auch und gerade für Westeuropa. Dort wurden nicht nur die größeren Einheitsstaaten seit Ende der 1970er Jahre einer substanziellen Dezentralisierung unterzogen (Frankreich, Großbritannien, Italien, Spanien); vielmehr fanden sich auch in den unitarischen Bundesstaaten des Kontinents Reformansätze, die als Versuche zur „(Wieder-)Belebung“ des Föderalismus gelten können (Deutschland, Österreich).

Fragt man nach den Gründen für diese länderübergreifenden Reformtendenzen, fällt zunächst ein Argument ins Auge, das sich schon bei den „Klassikern“ der Vergleichenden Regierungslehre findet: Demnach erscheint das Föderalismusprinzip für die Entwicklung der Demokratie insofern förderlich, als eine vertikale Aufteilung hoheitlicher Kompetenzen die Machtposition des Zentralstaates einschränkt und damit der Mehrheitsherrschaft inner-

1 Der vorliegende Beitrag bildet die erweiterte und aktualisierte Fassung eines Vortrags, der im Rahmen des Workshops „Föderale Gewaltenteilung und Demokratie“ der Sektion „Vergleichende Politikwissenschaft" der Deutschen Vereinigung für Politische Wissenschaft (DVPW) am 27. September 2006 gehalten wurde. Für hilfreiche Kommentare und Anregungen danke ich den Diskussionsteilnehmern sowie den Mitgliedern der ZParl-Redaktion. 